\title{
Dysfunctional Elimination Syndrome
}

National Cancer Institute

\section{Source}

National Cancer Institute. Dysfunctional Elimination Syndrome. NCI Thesaurus. Code

C123227.

Fecal constipation or impaction resulting in bowel and bladder dysfunction. 